'Fundação Oswaldo Cruz (Fiocruz), Escola Nacional de Saúde Pública Sergio Arouca (Ensp), Programa de Pós-graduação em Saúde Pública - Rio de Janeiro (RJ), Brasil. lidianesdutra@gmail.com

2 Fundação Oswaldo Cruz (Fiocruz), Escola Nacional de Saúde Pública Sergio Arouca (Ensp), Departamento de Direitos Humanos, Saúde e Diversidade Cultural (DIHS) - Rio de Janeiro (RJ), Brasil. aldopachecoferreira@gmail. com

\section{Associação entre malformações congênitas e a utilização de agrotóxicos em monoculturas no Paraná, Brasil}

\author{
Association between birth defects and the use of agrochemicals in \\ monocultures in the state of Paraná, Brazil
}

Lidiane Silva Dutra', Aldo Pacheco Ferreira ${ }^{2}$

RESUMO O objetivo deste artigo é analisar a associação entre o uso de agrotóxicos e as malformações congênitas em municípios com maior exposição aos agrotóxicos no estado do Paraná, Brasil, entre 1994 e 2014. Estudo de abordagem quantitativa, ecológico, conduzido com informações dos nascidos vivos (Sinasc/Ministério da Saúde), elaborando-se taxas de malformações ocorridas de 1994 a 2003 e de 2004 a 2014. Foi encontrada uma tendência crescente nas taxas de malformação congênita no estado do Paraná, com destaque aos municípios de Francisco Beltrão e Cascavel. Essas malformações congênitas podem ser advindas da exposição da população a agrotóxicos, sendo uma sinalização expressiva nos problemas de saúde pública.

PALAVRAS-CHAVE Agrotóxicos. Malformações congênitas. Exposição ambiental.

ABSTRACT This article aims to analyse the association between the use of pesticides and congenital malformations in cities with highest exposure to pesticides in the State of Paraná, Brazil, between 1994 and 2014. It is an ecological, quantitative approach study, conducted with live births information (Sinasc/Ministry of Health), generating malformations rates that have taken place in 1994-2003 and 2004-2014. A growing trend in congenital malformation rates was found in the state of Paraná, especially in the cities of Francisco Beltrão and Cascavel. These congenital malformations may be due to exposure of population to pesticides, and a significant signaling in public health problems.

KEYWORDS Agrochemicals. Congenital abnormalities. Environmental exposure. 


\section{Introdução}

O consumo crescente de agrotóxicos para o manejo da agricultura faz com que esses compostos tenham importância cada vez mais significativa para o campo da saúde pública devido a seus efeitos em médio e longo prazo na saúde da população (ROSA; PESSOA; RIGOTTO, 2011). Nos últimos dez anos, o mercado mundial de agrotóxicos cresceu 93\%, sendo que o mercado brasileiro teve um crescimento de $190 \%$; e dentre os estados brasileiros com maior consumo de agrotóxicos, destaca-se o estado do Paraná representando 14,3\% desse quantitativo (IBAMA, [2013?]).

Por definição legal, Decreto ${ }^{\circ}$ 4.074/2002, agrotóxicos e afins são os produtos e os agentes de processos físicos, químicos ou biológicos, destinados ao uso nos setores de produção, no armazenamento e beneficiamento de produtos agrícolas, nas pastagens, na proteção de florestas, nativas ou implantadas, e de outros ecossistemas e também de ambientes urbanos, hídricos e industriais, cuja finalidade visa alterar a composição da flora ou da fauna, a fim de preservá-las da ação danosa de seres vivos considerados nocivos (TOMYTA, 2005).

O emprego de agrotóxicos tem implicado uma série de problemas relacionados com a contaminação ambiental e com a saúde pública, pois eles dispersam-se no ambiente, contaminando a água, o solo e os alimentos, além de persistirem nas cadeias tróficas (ROSA; PESSOA; RIGOTTO, 2011). Estudos denotam que vários agrotóxicos podem afetar o sistema reprodutivo masculino de animais e também o desenvolvimento embriofetal após exposição intrauterina, dentre as quais, destacam-se as Malformações Congênitas (MC) (BENITEZ-LEITE; MACCHI; ACOSTA, 2009; RIGOTTO ET AL., 2013). Apesar de existirem múltiplos mecanismos que podem resultar na alteração da secreção das glândulas hormonais, destaca-se a participação dos disruptores endócrinos, compostos capazes de mimetizar hormônios verdadeiros devido a semelhanças entre as suas estruturas moleculares. Há uma grande quantidade de substâncias que são consideradas disruptoras endócrinas e, entre estas, estão presentes diversos agrotóxicos (HEeREN; TYLER; MANDEYA, 2003).

As MC afetam de 3\% a 5\% de todos os nascimentos, sendo que um terço desses defeitos põe em perigo a vida (BENITEZ-LEITE; MACCHI; ACOSTA, 2009). No Brasil e na América Latina, os óbitos por MC no primeiro ano de vida vêm crescendo, a exemplo do que acontece nos países desenvolvidos, e é hoje considerado de relevância para a saúde pública (RIGOTTO ET AL., 2013). Nesse contexto, a prevenção das MC e a investigação dos fatores de risco adquirem particular significado (CAVIERES, 2004; CALVERT ET AL., 2007; BENÍTEZ-LEITE; MACCHI; ACOSTA, 2009).

Ao considerar o modelo de desenvolvimento adotado na agricultura brasileira que se baseia na crescente demanda por substâncias químicas, estudos que analisem o impacto do uso dos agrotóxicos são relevantes para mensurar os desdobramentos nas populações atingidas. Assim, o presente estudo tem por objetivo analisar a distribuição temporal das MC observadas no estado do Paraná no período de 1994 a 2014, assim como avaliar a natureza da correlação existente entre a tendência observada e o volume de agrotóxicos tidos como disruptores endócrinos.

\section{Metodologia}

Trata-se de estudo de abordagem quantitativa, ecológico, conduzido com informações dos nascidos vivos, elaborando-se taxas de MC ocorridas de 1994 a 2003 e de 2004 a 2014.

As informações sobre os nascidos vivos foram obtidas do Sistema de Informação sobre Nascidos Vivos (Sinasc) do Ministério da Saúde (BRASIL, 2016). Este é um sistema de informação de base populacional que agrega os registros contidos na declaração de nascidos vivos, o que permite diversas análises na área de saúde materno-infantil. Os anos escolhidos compõem 
todo o sistema do banco de dados, que começou os registros em 1994 e os têm atualizados até 2014. Foram desconsiderados os casos de nascidos vivos com registro ignorado ou desconhecido. As taxas de MC foram calculadas por meio da seguinte fórmula:

Taxa de malformação congênita $=\underline{\mathrm{n}^{0} \text { de nascidos vivos com malformação } \times 1.000}$

Total de nascidos vivos no período

Foi feita uma comparação das taxas de MC ocorridas no estado do Paraná, dividindo em duas partes o período acima mencionado: primeiro período (1994-2003) e segundo período (2004-2014). As taxas referentes ao primeiro período foram tidas como referência, uma vez que apresentavam um menor nível de exposição. O risco relativo, comparando-se os períodos assumidos, foi calculado por meio do Odds Ratio e o Intervalo de Confiança (IC) adotado para as amostras foi de $95 \%$.

Para a construção da variável de exposição, levou-se em consideração a quantidade de lavouras plantadas por hec-tare de acordo com o ano de plantio para cada Unidade Regional (UR) selecionada e também para o estado do Paraná no período de 1994 a 2014. Selecionaram-se 4 grãos de cultivo, principais commodites agrícolas brasileiras, que correspondem a mais de $70 \%$ da produção do estado, sendo eles: algodão, cana-de-açúcar, milho e soja. Os dados sobre plantio foram obtidos por intermédio do Instituto Brasileiro de Geografia e Estatística (IBGE, 2016).

A relação dos agrotóxicos utilizados por princípio ativo para a média dos anos de 2014 a 2015 foi obtida por meio da Agência de Defesa Agropecuária do Paraná (Adapar). Esses dados somente estavam disponíveis para os anos supracitados. Apesar da variável de exposição estar pautada na produção de grãos pelo estado, esses dados dão subsídio a essa variável, uma vez que o tipo de monocultura não foi alterado. Assim sendo, pode-se supor que os ativos utilizados não variaram significativamente ao longo dos anos.

A Adapar processa as informações recebidas por meio do Sistema de Controle do Comércio e Uso de Agrotóxicos no Estado do Paraná (Siagro), pelo qual as empresas comerciantes declaram as vendas desses insumos para os produtores paranaenses. Os princípios ativos foram dispostos para um conjunto de municípios denominadas Unidades Regionais (URS), distribuídos de acordo com critério da referida agência, totalizando 20 URS que agrupavam os $399 \mathrm{mu}-$ nicípios do estado do Paraná.

Dos 262 princípios ativos presentes na listagem da Adapar, foram selecionados aqueles sabidamente reconhecidos como disruptores endócrinos, resultando em um total de 68 agrotóxicos (MNIF ET AL., 2011). Os princípios ativos selecionados foram divididos em 4 quartis e classificados de acordo com o consumo em: alto; médio; baixo e muito baixo. Os quartis referentes ao estado do Paraná possuíam 17 princípios ativos cada. Dos 68 agrotóxicos classificados como disruptores endócrinos, 55 estavam presentes nas URS analisadas. Assim, na distribuição desses ativos por quartis, os três primeiros possuíam 14 princípios ativos cada, e o último quartil possuía 13 princípios ativos.

Posteriormente, foi calculado o total de agrotóxicos consumidos em cada UR e foi feita a divisão delas em dois grupos, cada qual com um total de 10 URS distribuídas em: alto e baixo uso de agrotóxicos. Devido ao quantitativo de cidades diferentes nas URS, selecionaram-se duas delas que possuíam um número total de municípios próximo: Cascavel (C) e Francisco Beltrão (FB) que representam, respectivamente, URS com alto e baixo consumo de agrotóxicos. Essas duas URS foram comparadas quanto ao número de MC registradas no período de 1994 a 2014. Assim, foi feita uma análise espacial e 
temporal acerca dessas duas URS escolhidas.

A UR de Cascavel é composta pelos municípios: Anahy; Boa Vista da Aparecida; Braganey; Cafelândia; Campo Bonito; Capitão Leônidas Marques; Cascavel; Catanduvas; Céu Azul; Corbélia; Diamante d'Oeste; Foz do Iguaçu; Ibema; Iguatu; Itaipulândia; Lindoeste; Matelândia; Medianeira; Missal; Nova Aurora; Ramilândia; Santa Lúcia; Santa Tereza do Oeste; Santa Terezinha de Itaipu; São Miguel do Iguaçu; Serranópolis do Iguaçu; Três Barras do Paraná; Vera Cruz do Oeste.

A UR de Francisco Beltrão é composta pelos municípios: Ampére; Barracão; Bela Vista da Caroba; Boa Esperança do Iguaçu; Bom Jesus do Sul; Capanema; Cruzeiro do Iguaçu; Dois Vizinhos; Enéas Marques; Flor da Serra do Sul; Francisco Beltrão; Manfrinópolis; Marmeleiro; Nova Esperança do Sudoeste; Nova Prata do Iguaçu; Pérola d'Oeste; Pinhal de São Bento; Planalto; Pranchita; Realeza; Renascença; Salgado Filho; Salto do Lontra; Santa Izabel do Oeste; Santo Antônio do Sudoeste; São Jorge d'Oeste; Verê.
Para análise dos dados, foram construídas planilhas no Microsoft Excel ${ }^{\circledR}$. Foram formuladas tabelas de frequências simples (percentual), intervalo de confiança de $95 \%$ e razão de chances. As associações entre as variáveis independentes com a variável dependente foram estabelecidas utilizando-se os testes de razão de chances com o respectivo intervalo de confiança de $95 \%$ para as variáveis categóricas. Foi adotado o nível de significância de 5\% em todos os testes.

\section{Resultados e discussão}

A quantidade total de agrotóxicos utilizada em toneladas no estado do Paraná, de acordo com a UR, encontra-se no gráfico 1. As URS com maior consumo de agrotóxico (média 2014-2015) foram Cascavel (5.107,46 toneladas), Ponta Grossa (3.526,73 toneladas) e Toledo (3.336,95 toneladas). Das 20 URS apresentadas, 11 tiveram consumo de agrotóxicos acima de 1 tonelada.

Gráfico 1. Quantidade de agrotóxicos em toneladas (média 2014-2015) utilizada no estado do Paraná, por Unidades Regionais

Toneladas de Agrotóxicos (média 2014-2015)

6.000

5.000

4.000

3.000

2.000

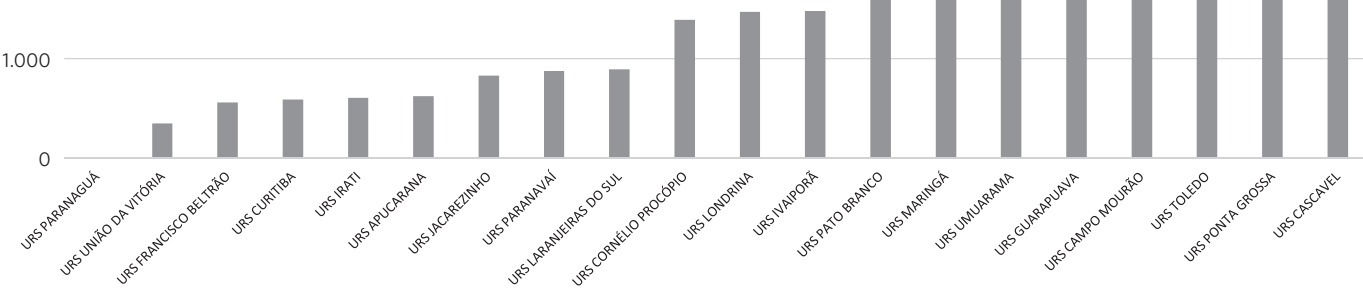

Fonte: Elaboração própria. 
Os princípios ativos dos agrotóxicos utilizados no estado do Paraná e nas URS Cascavel e Francisco Beltrão, com suas respectivas porcentagens para a média dos anos 2014/2015, encontram-se na tabela 1. As colorações observadas representam os quartis de distribuição dos princípios ativos em relação ao consumo, correspondendo do preto ao branco respectivamente a: alto, médio, baixo e muito baixo $\left(1^{\circ}, 2^{\circ}, 3^{\circ}\right.$ e $4^{\circ}$ quartis). Esses princípios ativos selecionados por serem sabidamente disruptores endócrinos representam 32,6\% (32.171,94 toneladas) do total de agrotóxicos consumido no estado (98.623,72 toneladas). A soma dos 5 princípios ativos com maior consumo, glifosato, atrazina, acefato, 2,4-D e epoxiconazol/ piraclostrobina, ultrapassam $50 \%$ do total de agrotóxicos utilizados.

Tabela 1. Princípios ativos e porcentagem referentes aos agrotóxicos utilizados no estado do Paraná e nas URS de Cascavel e Francisco Beltrão, 2015

\begin{tabular}{|c|c|c|c|}
\hline AGROTÓXICO (Princípio Ativo) & PR (\%) & URS C (\%) & URS FB (\%) \\
\hline Glifosato & 27,46 & 30,28 & 35,17 \\
\hline Atrazina & 7,41 & 9,80 & 4,27 \\
\hline Acefato & 7,35 & 6,71 & 5,15 \\
\hline $2,4-D$ & 5,99 & 7,18 & 5,57 \\
\hline Epoxiconazol/Piraclostrobina & 4,77 & 4,51 & 2,80 \\
\hline Metomil & 4,42 & 3,52 & 3,06 \\
\hline Ciproconazol/Picoxistrobina & 3,88 & 2,55 & 4,40 \\
\hline Glifosato/Equivalente ácido de Glifosato & 3,82 & 2,38 & 3,53 \\
\hline Diurom/Paraquate & 3,79 & 2,65 & 2,59 \\
\hline Carbendazim & 3,21 & 2,81 & 1,46 \\
\hline Cipermetrina & 3,02 & 4,03 & 2,00 \\
\hline Diflubenzurom & 2,81 & 4,06 & 1,49 \\
\hline Propiconazol & 2,80 & 3,27 & 3,34 \\
\hline Ciproconazol/Trifloxistrobina & 2,37 & 2,19 & 4,06 \\
\hline Tebuconazol/Trifloxistrobina & 1,87 & 2,87 & 2,06 \\
\hline Clorpirifós & 1,52 & 0,87 & 1,58 \\
\hline 2,4-D/PICLORAM & 1,21 & 0,56 & 1,22 \\
\hline Deltametrina & 1,07 & 1,14 & 0,62 \\
\hline Carbendazim/Tiram & 0,98 & 1,28 & 1,20 \\
\hline Metomil/METANOL & 0,96 & 1,08 & 1,93 \\
\hline Tebuconazol & 0,91 & 0,45 & 1,25 \\
\hline Iprodiona & 0,81 & 0,30 & 0,41 \\
\hline $\begin{array}{l}\text { 2,4-D/PICLORAM/Equivalente de Ácido de PICLORAM/Equivalente } \\
\text { ácido de 2,4-D }\end{array}$ & 0,80 & 1,23 & 1,65 \\
\hline 2,4-D/Equivalente ácido de 2,4-D & 0,69 & 1,14 & 1,52 \\
\hline Ciproconazol & 0,58 & 0,25 & 0,83 \\
\hline Atrazina/Simazina & 0,57 & 0,53 & 4,34 \\
\hline Tebuconazol/Picoxistrobina & $5,65 E-01$ & $2,42 \mathrm{E}-01$ & $2,50 E-01$ \\
\hline Diurom/Hexazinona & 4,97E-01 & $3,22 \mathrm{E}-04$ & -- \\
\hline Clorotalonil & $3,90 \mathrm{E}-01$ & 2,32E-02 & 8,07E-02 \\
\hline Procimidona & 3,36E-01 & $3,48 \mathrm{E}-02$ & $6,36 \mathrm{E}-03$ \\
\hline
\end{tabular}




\begin{tabular}{|c|c|c|c|}
\hline Carbendazim/Tebuconazol/CRESOXIM-METÍLICO & 2,97E-01 & $4,23 \mathrm{E}-01$ & 2,23E-01 \\
\hline Epoxiconazol/CRESOXIM-METÍLICO & $2,60 \mathrm{E}-01$ & $1,41 \mathrm{E}-01$ & $3,84 \mathrm{E}-01$ \\
\hline Dimetoato & 2,27E-01 & $5,15 \mathrm{E}-03$ & $1,19 \mathrm{E}-03$ \\
\hline Carbendazim/Flutriafol & 2,24E-01 & $1,64 \mathrm{E}-01$ & $1,11 \mathrm{E}-01$ \\
\hline Carbendazim/Tebuconazol & $1,95 \mathrm{E}-01$ & 4,00E-01 & $3,26 \mathrm{E}-01$ \\
\hline Carbofurano & $1,83 \mathrm{E}-01$ & $3,38 \mathrm{E}-02$ & $2,98 \mathrm{E}-02$ \\
\hline Flutriafol/Tiofanato-Metílico & $1,78 \mathrm{E}-01$ & $6,15 E-02$ & $1,63 \mathrm{E}-01$ \\
\hline Captana & $1,76 \mathrm{E}-01$ & $1,07 \mathrm{E}-01$ & $8,35 \mathrm{E}-03$ \\
\hline Piriproxifem & $1,58 \mathrm{E}-01$ & $1,48 \mathrm{E}-02$ & $3,18 \mathrm{E}-02$ \\
\hline Metribuzim & $1,52 \mathrm{E}-01$ & $2,25 \mathrm{E}-02$ & $3,18 \mathrm{E}-03$ \\
\hline Glufosinato - sal de amônio & $1,39 \mathrm{E}-01$ & $5,76 \mathrm{E}-02$ & $1,88 \mathrm{E}-01$ \\
\hline Propiconazol/Trifloxistrobina & $1,32 \mathrm{E}-01$ & -- & - \\
\hline Triadimenol & 1,19E-01 & $5,02 \mathrm{E}-02$ & $2,72 \mathrm{E}-01$ \\
\hline Flutriafol & $1,09 \mathrm{E}-01$ & 2,70E-02 & $7,75 \mathrm{E}-02$ \\
\hline Parationa-metílica & 1,07E-01 & $1,72 \mathrm{E}-01$ & $6,00 \mathrm{E}-02$ \\
\hline Epoxiconazol & 7,60E-02 & $6,44 \mathrm{E}-04$ & $6,88 \mathrm{E}-02$ \\
\hline Diurom & $7,06 \mathrm{E}-02$ & 8,37E-03 & $2,39 \mathrm{E}-03$ \\
\hline Malationa & $6,25 \mathrm{E}-02$ & $1,31 \mathrm{E}-01$ & 7,47E-02 \\
\hline Permetrina & $5,95 \mathrm{E}-02$ & $1,51 \mathrm{E}-01$ & 1,39E-02 \\
\hline 2,4-D/Aminopiralide & $5,48 \mathrm{E}-02$ & $1,71 \mathrm{E}-02$ & 4,89E-02 \\
\hline Linurom & 3,92E-02 & $2,41 \mathrm{E}-02$ & $2,78 \mathrm{E}-03$ \\
\hline Ciproconazol/Tiametoxam & $2,41 \mathrm{E}-02$ & 1,93E-03 & - \\
\hline Tebuconazol/CRESOXIM-METíLICO & 2,30E-02 & -- & $6,36 \mathrm{E}-03$ \\
\hline Atrazina/Metolacloro & $1,61 \mathrm{E}-02$ & 5,15E-03 & - \\
\hline Trifluralina & 1,37E-02 & 4,19E-03 & $3,98 \mathrm{E}-04$ \\
\hline Cipermetrina/Tiametoxam & $7,06 \mathrm{E}-03$ & $3,54 \mathrm{E}-03$ & $5,01 \mathrm{E}-02$ \\
\hline Cipermetrina/Profenofós & $7,05 \mathrm{E}-03$ & -- & - \\
\hline Diurom/Hexazinona/Sulfometurom Metílico & $6,66 \mathrm{E}-03$ & -- & - \\
\hline Fenitrotiona & $6,04 \mathrm{E}-03$ & -- & 8,35E-03 \\
\hline Fenarimol & $2,18 \mathrm{E}-03$ & -- & - \\
\hline Ciproconazol/Propiconazol & $8,70 \mathrm{E}-04$ & -- & 1,19E-03 \\
\hline Flutriafol/Imidacloprido & $5,49 \mathrm{E}-04$ & -- & - \\
\hline Glifosato/Imazetapir & $5,36 \mathrm{E}-04$ & -- & - \\
\hline Glifosato/S-METOLACLORO & $2,75 \mathrm{E}-04$ & -- & - \\
\hline Miclobutanil & $1,57 \mathrm{E}-04$ & -- & $3,98 \mathrm{E}-04$ \\
\hline Endossulfam & $5,11 \mathrm{E}-05$ & $3,22 \mathrm{E}-04$ & - \\
\hline Procloraz & $2,59 \mathrm{E}-05$ & -- & - \\
\hline Alacloro/Atrazina & $2,08 \mathrm{E}-05$ & -- & - \\
\hline Total & 68 & 55 & 55 \\
\hline
\end{tabular}

Fonte: Elaboração própria. 
O gráfico 2 apresenta a área plantada referente aos principais grãos produzidos no estado do Paraná e nas URS Cascavel e Francisco Beltrão, no período de 1994 a 2014. Entre 2000 e 2014, a área plantada para a produção de grãos no estado do Paraná aumentou em 39\% (5,9 mil hectares para 8,2 mil hectares) enquanto o consumo de agrotóxicos aumentou em 111\% (27,6 toneladas para 57,8 toneladas) (IBAMA, [2013?]; |BGE, 2016). Esses dados demonstram que a proporção entre área plantada e consumo de agrotóxicos é diferente, sendo esta última significativamente maior que a primeira. Baseado nisto, o gráfico 2 corrobora a hipótese de que a exposição ambiental sofrida pela população tem aumentado ao longo do tempo. Apesar do quantitativo de agrotóxicos não discriminar os princípios ativos utilizados, pode-se supor que eles não variaram significativamente, uma vez que as culturas de grãos continuam a ser as mesmas.

Gráfico 2. Quantitativo de hectares plantados na produção de grãos no estado do Paraná, da URS de Cascavel e Francisco Beltrão, 1994-2014

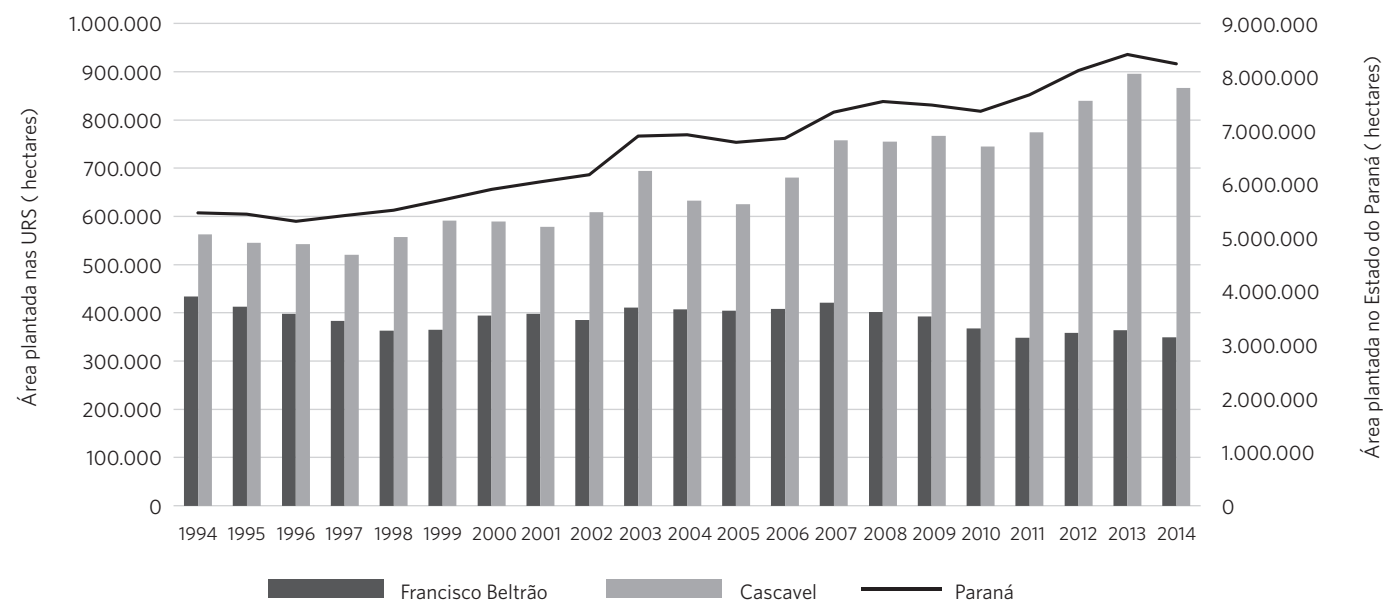

Fonte: Elaboração própria.

Houve maior taxa de MC para a UR Cascavel e para o estado do Paraná no período de 2003 a 2014. Foram encontradas associações positivas entre exposição aos agrotóxicos e MC, porém, para algumas delas, a associação não foi estatisticamente significativa. A tabela 2 expressa a Odds Ratios bruta e intervalos de confiança de nascidos vivos com MC para todo o estado do Paraná e para as URS Cascavel e Francisco Beltrão, entre 1994 e 2014.

As taxas referentes ao estado do Paraná, como um todo, são maiores que as encontradas na UR de maior exposição. É importante ressaltar que as análises das URS se referem a um total de 55 municípios, sendo esta uma parcela $(13,8 \%)$ do total (399) de municípios do estado. Também é relevante dizer que, se todo o estado fosse analisado por intermédio das URS apresentadas pela Adapar, haveria mais 193 municípios classificados em 'alto consumo de agrotóxicos' e 151 municípios classificados em 'baixo consumo de agrotóxicos'. Assim, os dados referentes ao estado demonstram que, ao longo do tempo, o agronegócio tem avançado e, apesar de haver regiões mais ou menos agrárias presentes no estado, a contaminação da população aumenta como um todo, demonstrando que as fronteiras agrícolas e os desdobramentos referentes a ela estão cada vez mais próximos de centros urbanos, seja por meio de uma aproximação literalmente física ou por meio dos contaminantes existentes na água, no ar ou nos alimentos ingeridos por essa população. 
Tabela 2. Odds Ratios brutos e intervalos de confiança de nascidos vivos com malformação congênita para variáveis controle para todo o estado do Paraná e para as URS de Cascavel e Francisco Beltrão, 1994-2014

\begin{tabular}{|c|c|c|c|c|c|c|c|c|}
\hline \multirow{2}{*}{ Malformações Congênitas } & \multicolumn{4}{|c|}{ Paraná } & \multicolumn{4}{|c|}{ URS Fernando Beltrão (FB) e Cascavel (C) } \\
\hline & $1994-2003$ & 2004-2014 & OR & IC95\% & FB & C & OR & IC95\% \\
\hline Espinha bífida & 141 & 346 & 2,66 & $2,18-3,23$ & 15 & 60 & 1,43 & $0,81-2,52$ \\
\hline $\begin{array}{l}\text { Outras malformações congênitas do sistema } \\
\text { nervoso }\end{array}$ & 572 & 1218 & 2,31 & $2,09-2,55$ & 59 & 192 & 1,16 & $0,87-1,56$ \\
\hline $\begin{array}{l}\text { Malformações congênitas do aparelho circula- } \\
\text { tório }\end{array}$ & 159 & 781 & 5,32 & $4,48-6,31$ & 37 & 114 & 1,10 & $0,76-1,60$ \\
\hline Fenda labial e fenda palatina & 488 & 1160 & 2,57 & $2,32-2,86$ & 39 & 177 & 1,62 & $1,15-2,30$ \\
\hline $\begin{array}{l}\text { Ausência atresia e estenose do intestino del- } \\
\text { gado }\end{array}$ & 12 & 24 & 2,17 & $1,08-4,33$ & 1 & 5 & 1,79 & $0,21-15,31$ \\
\hline $\begin{array}{l}\text { Outras malformações congênitas aparelho } \\
\text { digestivo }\end{array}$ & 217 & 592 & 2,95 & $2,53-3,45$ & 27 & 95 & 1,26 & $0,82-1,93$ \\
\hline Testículo não descido & 15 & 92 & 6,64 & $3,85-11,46$ & 2 & 13 & 2,33 & $0,52-10,30$ \\
\hline Outras malformações do aparelho geniturinário & 281 & 850 & 3,28 & $2,86-3,75$ & 40 & 145 & 1,30 & $0,91-1,84$ \\
\hline Deformidades congênitas do quadril & 43 & 56 & 1,41 & $0,95-2,10$ & 3 & 8 & 0,95 & $0,25-3,60$ \\
\hline Deformidades congênitas dos pés & 653 & 1589 & 2,63 & $2,41-2,89$ & 59 & 282 & 1,71 & $1,29-2,26$ \\
\hline $\begin{array}{l}\text { Outras malformações e deformações congênitas } \\
\text { do aparelho osteomuscular }\end{array}$ & 843 & 2873 & 3,69 & $3,42-3,98$ & 76 & 408 & 2,70 & $2,03-3,59$ \\
\hline Outras malformações congênitas & 450 & 1325 & 3,19 & $2,86-3,55$ & 54 & 208 & 1,38 & $1,02-1,86$ \\
\hline Anomalias cromossômicas NCOP* & 332 & 837 & 2,73 & $2,40-3,10$ & 38 & 104 & 0,98 & $0,68-1,42$ \\
\hline Hemangioma e linfangioma & 32 & 44 & 1,49 & $0,94-2,35$ & 3 & 9 & 1,07 & $0,29-3,96$ \\
\hline $\begin{array}{l}\text { Total de nascidos vivos com malformações } \\
\text { congênitas }\end{array}$ & 4238 & 11787 & 3,01 & $2,91-3,12$ & 453 & 1820 & 1,44 & $1,30-1,59$ \\
\hline
\end{tabular}

Fonte: Elaboração própria.

*NCOP: não classificadas em outra parte. Odds Ratio - OR; Intervalo de Confiança - IC.

Alguns estudos evidenciam maior ocorrência de $\mathrm{MC}$ devido à proximidade das residências às áreas de cultivo (RULL; RITZ; SHAW, 2006; BRENDER ET AL., 2010). As fronteiras urbano-rurais estão cada dia mais próximas, o que pode propiciar a exposição de moradores periurbanos à contaminação de agrotóxicos (BRENDER ET AL., 2010). Além disso, a contaminação do ar e a consequente circulação deste também pode ser vista como fonte de exposição.

Outra fonte de exposição é a água consumida pela população. As técnicas necessárias à remoção de contaminantes orgânicos em água correspondem a tecnologias pouco comuns à maioria das estações de tratamento de água convencionais, como adsorção em carvão ativado e filtração por membranas (osmose reversa e nanofiltração), o que evidencia o risco de que tais substâncias passem desapercebidas durante os processos de tratamento, colocando em risco a saúde da população. Além disso, somente um pequeno número dos agrotóxicos utilizados no País têm limites de resíduos presentes na legislação brasileira, e o monitoramento destes contaminantes por parte dos prestadores de serviços de abastecimento e pelo setor saúde fica muito aquém do desejado, sendo incompleto ou ausente em vários casos (FERNANDES NETO; SARCINELLI, 2009).

Diversos compostos agrícolas, tais como atrazina e nitrato, têm sido encontrados em fontes de água potável em todo o mundo, tanto isolados como na forma de misturas (JAIPIEAM ET AL., 2009). O estudo de Toccalino, Norman e Scott (2012) evidenciou a presença da mistura desses contaminantes em 383 poços públicos de distribuição de água, distribuídos entre 
35 estados norteamericanos. Esse fato deve ser mencionado com particular relevância, pois as substâncias misturadas podem reagir e formar compostos com características químicas mais tóxicas e prejudiciais que os reagentes dos quais se originaram. Em um estudo recente com embriões de galinha expostas a $\mathrm{N}$-nitrosoatrazina (um composto $\mathrm{N}$-nitrosamina derivado da reação entre o nitrito e atrazina), Joshi et al. (2013) observaram malformações em $23 \%$ dos embriões, incluindo coração, defeitos do tubo neural, gastrosquise e outros defeitos. Apesar do estudo acima estar relacionado com um modelo experimental animal, deve-se salientar que a atrazina está entre os agrotóxicos com maior utilização pelo estado do Paraná (7,41\% correspondente a $2.384,00$ toneladas), o que dá maior relevância a esses dados.

A maior associação encontrada para o estado do Paraná foi referente à malformação classificada como 'Testículo não descido'. Por serem disruptores endócrinos, muitos agrotóxicos são suspeitos de influenciar a diferenciação sexual do feto e outros desfechos dependentes de hormônios sexuais. Há evidências sobre a associação entre criptorquidia, (SILVA ET AL., 2011) hipospadia (BAY ET AL., 2006) e a exposição a agrotóxicos, ressaltando que tais problemas são relacionados com a flutuação de hormônios femininos e masculinos no período gestacional, e estes, por sua vez, são influenciados diretamente pelas condições ambientais.

A segunda maior associação encontrada para o estado do Paraná foi referente às 'Malformações congênitas do aparelho circulatório' $(\mathrm{OR}=5,32, \mathrm{IC} 95 \%=4,48-6,31)$. Um estudo de caso-controle analisou a incidência de diferentes tipos de malformações cardíacas e a exposição à agrotóxicos específicos devido à proximidade da residência dos indivíduos a áreas de plantação. Foram encontradas associações entre MC e agrotóxicos, respectivamente, entre: tetralogia de Fallot e o neocotinóide imidacloprida; síndrome da hipoplasia do coração esquerdo e o fungicida azoxistrobina; estenose pulmonar valvar e os herbicidas norflurazon, 2,4-D e paraquat; defeito do septo ventricular perimembranoso e o acaricida abamectin; defeito de septo atrial e hexazinona, o herbicida 2,4-D, o acaricida óxido de fembutatina e os inseticidas clorpirifós e lambda-cialotrina (CARMichael et Al., 2014). Outro estudo caso-controle também encontrou associação entre MC cardíacas e a exposição ocupacional das mães a classes específicas de agrotóxicos, sendo estas respectivamente: defeito de septo atrial e inseticidas; síndrome da hipoplasia do coração esquerdo e inseticidas e herbicidas; tetralogia de Fallot e estenose da válvula pulmonar e fungicidas, herbicidas e inseticidas (ROCHELEAU ET AL., 2015).

A terceira maior associação encontrada entre as URS analisadas foi referente às malformações de 'fenda labial e fenda palatina' $(\mathrm{OR}=1,62$, IC95\%= 1,15 - 2,30). Três estudos demonstraram a associação entre a exposição a agrotóxicos e Defeitos no Tubo Neural (DTN). O primeiro evidenciou o aumento no risco destas $\mathrm{MC}$ e a proximidade da residência materna a menos de $1 \mathrm{~km}$ das áreas de aplicação de agrotóxicos. Associações positivas e estatisticamente significativas foram encontradas para: espinha bífida e outros DTN e agrotóxicos quimicamente classificados como amidas, benzimidazóis e metil carbamatos; e anencefalia associada aos organofosforados (RULL; RITZ; SHAW, 2006). O segundo estudo constatou que agrotóxicos específicos estavam relacionados com malformações específicas: anencefalia foi relacionada a 2,4-D, metomil, imidacloprida e ao éster fosfato $\alpha$-(para-nonil fenol)- $\omega$-hidroxi polioxietileno; espinha bífida a bromoxinil; fenda labial e palatina a trifluralina e maneb (YANG ET AL., 2014). O terceiro estudo observou o aumento do risco de DTN e espinha bífida para exposição à inseticidas e herbicidas. Também foi vista associação entre encefalocele e anencefalia e exposição cumulativa a herbicidas, inseticidas e fungicidas (MAKELARSKI ET AL., 2014). Vários dos agrotóxicos 
mencionados nesses estudos também estão presentes na tabela 1, o que evidencia o potencial danoso dessas substâncias.

Vieses importantes de serem mencionados neste estudo são os óbitos fetais e os abortos espontâneos. Regidor et al. (2004) demonstraram que famílias de agricultores tiveram maior risco de apresentarem gestações com desfecho em morte fetal em áreas onde os agrotóxicos são mais utilizados quando comparadas a outras regiões da Espanha, sendo o risco ainda maior quando o período da concepção coincide com o máximo uso dos agrotóxicos. Um estudo italiano verificou a presença de agrotóxicos disruptores endócrinos em 11 em um total de 24 natimortos, incluindo agrotóxicos organoclorados e organofosforados como clordano, heptacloro, clorfenvinfós, clorpirifós, e ainda aqueles cujo uso está banido como DDT e seu metabólito DDE (RONCATI; PISCIOLl; PUSIOL, 2016). Pode-se inferir que muitos casos de MC resultaram em óbitos fetais e abortos espontâneos, o que tornaria os números relacionados com o desfecho estudado ainda maiores. Análises mais aprofundadas acerca das causas das MC, como investigação genética, ajudariam a estabelecer com maior precisão os fatores ambientais envolvidos.

\section{Conclusões}

O presente estudo encontrou uma taxa maior de malformação congênita para a UR com maior uso de agrotóxico (UR Cascavel) e para o estado no Paraná no período com maior uso de agrotóxicos (2004-2014). A análise espacial proposta neste artigo levou em conta uma pequena parte do estado do
Paraná, as URS escolhidas, sendo válidas as afirmações para este quantitativo de cidades. A análise temporal, no entanto, cabe a todo o estado. As tendências crescentes nessas taxas sugerem maior exposição ambiental à população dos municípios envolvidos e de toda a população do estado do Paraná ao longo do tempo.

Além de todos os problemas já citados, o controle efetivo da exposição a esses pesticidas é muito pequeno e escasso no cenário brasileiro. Os dados referentes ao uso dos produtos não são sistematizados em bancos de dados informatizados para a grande maioria dos estados do País. Isso dificulta a mensuração do impacto da exposição ambiental desses produtos sofrida pela população. Além disso, o lobby exercido pelas grandes corporações impede, quase sempre, o acesso à informação.

Muitas são as dificuldades no estabelecimento da relação entre MC e a exposição a agrotóxicos, a despeito de se ter substâncias reconhecidamente disruptoras endócrinas presentes nesses químicos. Apesar de diversos entraves metodológicos, esses estudos subsidiam pressupostos acerca da exposição ambiental a esses contaminantes, e o aumento significativo nas taxas de $\mathrm{MC}$ nos municípios que fazem uso intensivo de agrotóxicos e de outros municípios com menor uso destes insumos agrícolas corroboram essas hipóteses.

\section{Agradecimentos}

Agradecemos aos profissionais da Adapar que solicitamente nos forneceram os dados para que esta pesquisa fosse realizada. 


\section{Referências}

BAY, K. et al. Testicular digenesis syndrome: possible role of endocrine disrupters. Best Practice \& Research Clinical Endocrinology \& Metabolism, Amsterdam, v. 20, n. 1, p. 77-90, mar. 2006.

BENÍTEZ-LEITE, S.; MACCHI, M. L.; ACOSTA, M. Malformaciones congénitas associadas a agrotóxicos. Revista de la Sociedad Boliviana de Pediatría, Sucre, v. 48, n. 3, p. 204-217, 2009. Disponível em: <http://www. scielo.edu.uy/pdf/adp/v80n3/v80n3al2.pdf >. Acesso em: 7 jul. 2016.

BRASIL. Decreto no 4.074, de 4 de janeiro de 2002. Regulamenta a Lei $n^{\circ} 7.802$, de 11 de julho de 1989, que dispõe sobre a pesquisa, a experimentação, a produção, a embalagem e rotulagem, o transporte, o armazenamento, a comercialização, a propaganda comercial, a utilização, a importação, a exportação, o destino final dos resíduos e embalagens, o registro, a classificação, o controle, a inspeção e a fiscalização de agrotóxicos, seus componentes e afins, e dá outras providências. Diário Oficial [da] União, Poder Executivo, Brasília, DF, 2002. Disponível em: <http://www.mma.gov.br/port/ conama/legiabre.cfm? codlegi=515 > . Acesso em: 27 jul. 2016.

Ministério da Saúde. DATASUS: Tecnologia da informação a serviço do SUS. Rio de Janeiro, 2016. Disponível em: <http://tabnet.datasus.gov.br/cgi/deftohtm.exe?sinasc/cnv/nvCE.def $>$. Acesso em: 13 maio 2016.

BRENDER, J. D. et al. Maternal pesticide exposure and neural tube defects in Mexican Americans. Annals of Epidemiology, Amsterdam, v. 20, n. 1, p. 16-22, 2010. Disponível em: <http://www.annalsofepidemiology. org/article/S1047-2797(09)00327-5/abstract?rss=yes>. Acesso em: 27 jul. 2016.

CALVERT, G. M. et al. Case Report: Three farmworkers who gave birth to infants with birth defects closely grouped in time and Place-Florida and North Carolina, 2004-2005. Environmental Health Perspectives, Bethesda, USA, v. 115, n. 5, p. 787-791, maio 2007. Disponível em: <https://www.ncbi.nlm.nih.gov/
pmc/articles/PMC1867963/>. Acesso em: 27 jul. 2016.

CARMICHAEL, S. L. et al. Residential agricultural pesticide exposures and risk of selected congenital heart defects among offspring in the San Joaquin Valley of California. Environmental Research, Bethesda, USA, v. 135, p. 133-138, nov. 2014. Disponível em: < https://www. ncbi.nlm.nih.gov/pubmed/25262086>. Acesso em: 27 jul. 2016.

CAVIERES, M. F. Exposición a pesticidas y toxicidad reproductiva y del desarrollo em humanos. Análisis de la evidencia epidemiológica y experimental. Revista Médica de Chile, Santiago, v. 132, p. 873-879, 2004. Disponível em: <http://www.scielo.cl/pdf/rmc/v132n7/ art14.pdf $>$. Acesso em: 27 jul. 2016.

FERNANDES NETO, M. L.; SARCINELLI, P. N. Agrotóxicos em água para consumo humano: uma abordagem de avaliação de risco e contribuição o processo de atualização da legislação brasileira. Engenharia Sanitaria e Ambiental, Rio de Janeiro, v. 14, p. 69-78, 2009. Disponível em: < http://www.abes-dn. org.br/publicacoes/engenharia/resaonline/v14n01/ RESA_vl4n1_p69-78.pdf>. Acesso em: 9 jul. 2016.

HEEREN, G. A.; TYLER, J.; MANDEYA, A. Agricultural chemical exposures and birth defects in the Eastern Cape Province, South Africa a case control study. Environmental Health, Londres, v. 2, n. 11, 2003. Disponível em: <https://ehjournal.biomedcentral.com/ articles/10.1186/1476-069X-2-11>. Acesso em: 9 jul. 2016.

\section{INSTITUTO BRASILEIRO DO MEIO AMBIENTE E} DOS RECURSOS NATURAIS RENOVÁVEIS (IBAMA). Boletim de Comercialização de Agrotóxicos e Afins - histórico de vendas de 2000 a 2012. Brasília, DF: IBAMA; MMA, [2013?]. Disponível em: <http://www.ibama.gov. br/servicosonline/index.php/difusao-de-conhecimento/relatorio-de-agrotoxicos/124-agrotoxicos-relatorio-semestral>. Acesso em: 23 jun. 2016.

INSTITUTO BRASILEIRO DE GEOGRAFIA E ESTATÍSTICA (IBGE). Tabela 1612: área plantada, 
área colhida, quantidade produzida, rendimento médio e valor da produção das lavouras temporárias. Rio de Janeiro, 2016. Disponível em: < http://www.sidra. ibge.gov.br/bda/tabela/listabl.asp?c $=1612 \& \mathrm{z}=\mathrm{t} \& \mathrm{o}=11>$. Acesso em: 7 jul. 2016.

JAIPIEAM, S. et al. Organophosphate pesticide residues in drinking water from artesian wells and health risk assessment of agricultural communities, Thailand. Human and ecological risk assessment, [s. 1.], v. 15, n. 6, p. 1304-1316, 2009. Disponível em: < https://www.ncbi. nlm.nih.gov/pmc/articles/PMC2871340/>. Acesso em: 8 jul. 2016.

JOSHI, N. et al. Developmental abnormalities in chicken embryos exposed to N-nitrosoatrazine. Journal of Toxicology and Environmental Health, [s. 1.], v. 76, n. 17, p. 1015-1022, 2013. Disponível em: <http://www.tandfonline.com/doi/abs/10.1080/15287394.2013.831721>. Acesso em: 8 jul. 2016.

MAKELARSKI, J. A. et al. Maternal periconceptional occupational pesticide exposure and neural tube defects. Birth Defects Research Clinical and Molecular Teratology, [s. 1.], v. 100, n. 11, p. 877-886, ago. 2015. Disponível em: <https://www.ncbi.nlm.nih.gov/pubmed/25124525>. Acesso em: 8 jul. 2016.

MNIF, W. et al. Effect of endocrine disruptor pesticides: a review. International Journal of Environmental Research and Public Health, Basel, v. 8, n. 6, p. 22652303, jun. 2011. Disponível em: <https://www.ncbi.nlm. nih.gov/pmc/articles/PMC3138025/>. Acesso em: 8 jul. 2016

QUEIROZ, E. K. R.; WAISSMANN, W. Occupational exposure and effects on the male reproductive system. Cadernos de Saúde Pública, Rio de Janeiro, v. 22, p. 485493, 2006. Disponível em: <http://www.scielo.br/pdf/ csp/v22n3/03.pdf>. Acesso em: 8 jul. 2016.

REGIDOR, E. et al. Paternal exposure to agricultural pesticides and cause specific fetal death. Occupational and Environmental Medicine, Londres, v. 61, n. 4, p. 334 339, abr. 2004. Disponível em: <https://www.ncbi.nlm. nih.gov/pmc/articles/PMC1740756/pdf/v061p00334. pdf $>$. Acesso em: 8 jul. 2016.
RIGOTTO, R. M. et al. Tendências de agravos crônicos à saúde associados a agrotóxicos em região de fruticultura no Ceará, Brasil. Revista Brasileira de Epidemiologia, Rio de Janeiro, v. 16, n. 3, p. 763-773, 2013.

ROCHELEAU, C. M. et al. Maternal occupational pesticide exposure and risk of congenital heart defects in the National Birth Defects Prevention Study. Birth Defects Research Clinical and Molecular Teratology, [s. 1.], n. 103, v. 10, p. 823-33, out. 2015.

RONCATI, L.; PISCIOLI, F.; PUSIOL, T. The endocrine disruptors among the environmental risk factors for stillbirth. Science of The Total Environment, v. 563-564, p. 1086-1087, 1 set. 2016.

\section{ROSA, I. F.; PESSOA, V. M.; RIGOTTO, R. M.}

Introdução: agrotóxicos, saúde humana e os caminhos do estudo epidemiológico. In: RIGOTTO, R. M. (Org.). Agrotóxicos, Trabalho e Saúde: vulnerabilidade e resistência no contexto da modernização agrícola no Baixo Jaguaribe/CE. Fortaleza: Edições UFC, 2011. Disponível em: <http://www.tramas.ufc.br/wp-content/uploads/2013/11/PARTE-01_Agrot\%C3\%B3xicosTrabalho-e-Sa\%C3\%BAde.pdf>. Acesso em: 8 jul. 2016.

RULL, R. P.; RITZ, B.; SHAW, G. M. Neural tube defects and maternal residential proximity to agricultural pesticide applications. American Journal of Epidemiology, Oxford, v. 163, n. 8, p. 743-753, 2006. Disponível em: < https://academic.oup.com/aje/ article/163/8/743/104675/Neural-Tube-Defects-andMaternal-Residential>. Acesso em: 8 jul. 2016.

SILVA, S. R. G. et al. Defeitos congênitos e exposição a agrotóxicos no Vale do São Francisco. Revista Brasileira de Ginecologia e Obstetrícia, Rio de Janeiro, v. 33, n. 1, p. 20-26, 2011. Disponível em: <http://www.scielo.br/pdf/ rbgo/v33nl/a03v33nl.pdf>. Acesso em: 8 jul. 2016.

TOCCALINO, P. L.; NORMAN, J. E.; SCOTT, J. C. Chemical mixtures in untreated water from public-supply wells in the U.S.: occurrence, composition, and potential toxicity. The Science of the total environment, v. 1, n. 431, p. 262-270, ago. 2012. Disponível em: <https://ca.water.usgs.gov/pubs/ 
Toccalino_Patricia_Journal_Article.pdf $>$. Acesso em: 8 jul. 2016.

TOMYTA, R.Y. Legislação de agrotóxicos e sua contribuição para a proteção da qualidade do meio ambiente. São Paulo: Roca, 2005.

YANG, W. et al. Residential agricultural pesticide exposures and risk of neural tube defects and orofacial clefts among offspring in the San Joaquin Valley of California. American Journal of Epidemiology, Oxford, v. 179, n. 6, p. 740-748, mar.
2014. Disponível em: <https://www.researchgate.net/ publication/260271212_Residential_Agricultural_ Pesticide_Exposures_and_Risk_of_Neural_Tube_ Defects_and_Orofacial_Clefts_Among_Offspring_in the_San_Joaquin_Valley_of_California>. Acesso em: 8 jul. 2016.

Recebido para publicação em agosto de 2016

Versão final em dezembro de 2016

Conflito de interesses: inexistente

Suporte financeiro: não houve 